\title{
QUASI-HOMOGENOUS MODEL OF ELECTROCHEMICAL MACHINING OF TURBINE ENGINE PARTS
}

\author{
Jerzy Kozak \\ Eukasiewicz Research Network - Institute of Aviation \\ Krakowska Av. 110/114, 02-256 Warsaw, Poland \\ tel.: +48228460011, fax: +48228464432 \\ e-mail: jerzy.kozak@ilot.edu.pl
}

\begin{abstract}
In order to increase the efficiency of jet engines hard to machine nickel-based and titanium-based alloys are in common use for aero engine components such as blades and blade integrated disks (BLISK). Electrochemical Machining (ECM) provides an economical and effective method for machining high strength and heat-resistant materials into complex shapes with high material removal rate without tool wear and without inducing residual stress. This article presents the physical and mathematical models of electrochemical shaping used in the manufacture of turbine engine parts. The modelling is based on the assumption that the multi-phase mixture filling the gap is treated as two-phase quasi-homogenous medium. The model describes the workpiece shape evolution in time, distribution the local gap size, flow parameters such as the static pressure and the velocity, temperature and void fraction as result of gas generation. The major features of the numerical computer program are briefly described with a selected example of machining a typical turbine blade. The results of computer simulation of effects of setting parameters ECM on accuracy-machined profile are discussed. The improvement of accuracy has been reached by using described sequence of ECM and Pulse ECM processes.
\end{abstract}

Keywords: electrochemical shaping, modelling, simulation, pulse current

\section{Introduction}

The long term strategic objectives "Flightpath 2050" have taken the Advisory Council for Aviation Research in Europe (ACARE) development targets beyond the year 2020. By year 2050, the following goals for air traffic have to be achieved [3]:

- $75 \%$ reduction in $\mathrm{CO}_{2}$ per passenger kilometre,

- $90 \%$ reduction in NOx emissions,

- $65 \%$ reduction in noise.

In particular, this will require improvement in aircraft engines. The further development of turbine engines is decreasing unitary fuel consumption, device weight, exploitation costs and negative effects on natural environment. The most important factors, which improve total engine efficiency, are increasing of the turbine inlet gas temperature increasing.

Therefore, the temperature increasing is one of the main directions of modern engine development. Advanced aero engine materials are developed to meet high strength level at elevated temperatures, to support higher compression rates and higher gas temperatures. Machining of these materials and complex components is really too difficult by mechanical methods.

The unconventional manufacturing technology such as Electrochemical Machining (ECM) is therefore be a cost-effective alternative for these materials and is still important in the manufacture of turbine engine parts.

As shown in Fig. 1 electrochemical machining is based on controlled anodic dissolution process in which the workpiece is the anode and the tool is the cathode of an electrolytic cell. In the ECM process, a low voltage $(8-30 \mathrm{~V})$ is normally applied between electrodes with a small gap size (usually 0.2 to $0.8 \mathrm{~mm}$ ) producing a high current density of the order of $\left(10\right.$ to $\left.100 \mathrm{~A} / \mathrm{cm}^{2}\right)$, and a metal removing rate ranging from an order $0.1 \mathrm{~mm} / \mathrm{min}$, to $10 \mathrm{~mm} / \mathrm{min}$. Electrolyte (typically 
$\mathrm{NaCl}$ or $\mathrm{NaNO}_{3}$ aqueous solutions) is supplied to flow through the gap with a velocity of 10 to $50 \mathrm{~m} / \mathrm{s}$ to maintain the electrochemical dissolution with high rate and to flush away the reactions products (usually gases and hydroxides) and heat generated caused by the passage of current and electrochemical reactions.

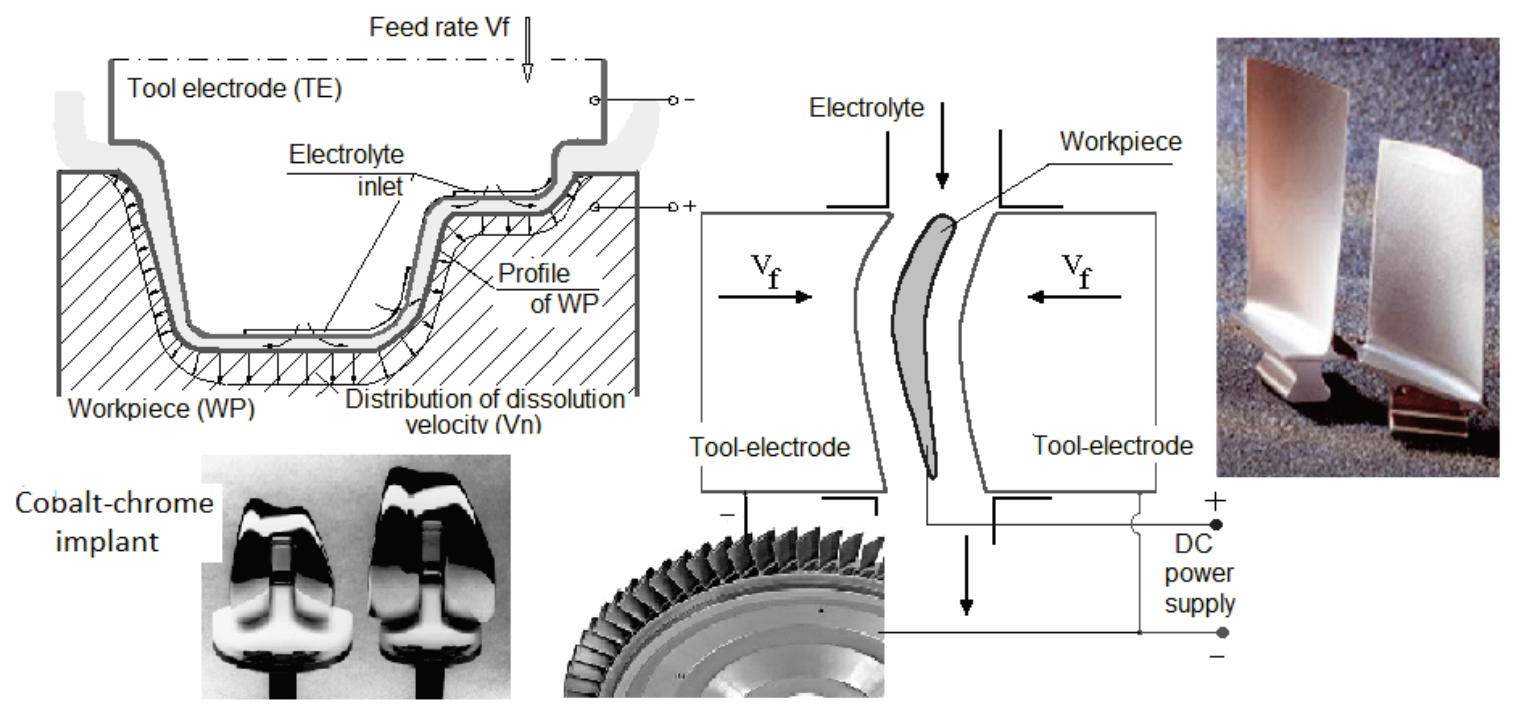

Fig. 1. Principal scheme of electrochemical machining (ECM) and examples of the machined details

As electrochemical dissolution proceeds, the tool electrode-cathode can be fed mechanically towards the workpiece - anode in order to maintain the machining action. Under these conditions, the inter-electrode gap width gradually tends to a steady-state value, and a shape, complementary to that of the cathode-tool, is reproduced approximately on the anode-workpiece. Being a nonmechanical metal removal process ECM is capable of machining any electrically conductive material with high stock removal rates regardless of their mechanical properties, such as hardness, elasticity and brittleness. It has been applied in diverse industries such as aerospace, automotive and electronics to manufacture airfoils and turbine blades, die and mould, artillery projectiles, surgical implants and prostheses, etc. [1, 2, 4-10].

Industrial practices in ECM have revealed some problems impeding its further development and wider acceptance by industrial users. Among them, prediction and control of the local gap width distribution (and hence, the control of dimensional accuracy), along with the design of tool electrodes for complex workpiece shapes and optimization of process, are the major problems encountered by ECM users.

The main objective of ECM is to achieve the required shape of workpiece within a given tolerance on the shape and dimensions. The tasks relating to this purpose can be reduced directly (or indirectly) to a problem of searching for a boundary of the area within which the machining, i.e. to a value boundary problems (moving boundary problem, free boundary problem or inverse boundary problem). Depending on which the electrode surface is to be determined all tasks can be divided into two groups:

1) tasks in which for a known shape of the tool electrode and known condition of machining, the evolution of a shape of workpiece surface has to be determined,

2) tasks in which the tool electrode shape is searched for, which ensures obtaining the required shape of the workpiece.

The first category of the problems is encountered in the analysis of ECM accuracy. The tasks from the second category mainly deal with the tool electrode design and most frequently are encountered in practice.

The article presents the physical and mathematical models, basis of which a process simulation software of has been developed. The example of results of computer simulation of blade profile 
shaping is discussed. In last section of the accuracy improvement by using pulse current is described.

\section{The brief overview of conditions in the gap between the electrodes}

During the ECM process, the generation of Joule heat and the liberation of hydrogen gas bubbles on the cathode surface accompany the release anodic dissolution products. Consequently, the electrolyte flowing in the gap becomes a multi-phase medium with rising temperature $T$ and void fraction in the flow direction, leading to corresponding changes in the electrical conductivity $\kappa$ and the current density $i$ along the gap as well. Simultaneously, the changes of other properties of medium such as specific medium density, viscosity, lead to redistribution of flow velocity $w$ and pressure $p$ in the gap. At the beginning of the ECM in the entire region of the gap, the hydrogen generated appears as a two-phase layer with changing of thickness in time and along flow. It led to difficulties in analysis of ECM process.

Relations between main factors occurring during ECM are shown schematically in Fig. 2 and Fig. 3. Analysis of these figures shows the complex nature of the machining process and mathematical difficulties in solving of shaping problems. All physical fields in the gap are nonstationary during whole time of machining. Since properties of electrolyte such as electrical conductivity $\kappa$ are depending on temperature $T$, and gas phase concentration $\beta$, which distributions depend on flow velocity $w$, and pressure fields $p$, as well as on current density $i$. The ECM processes have to be described by set of mass, heat and electric charge transfer equations.

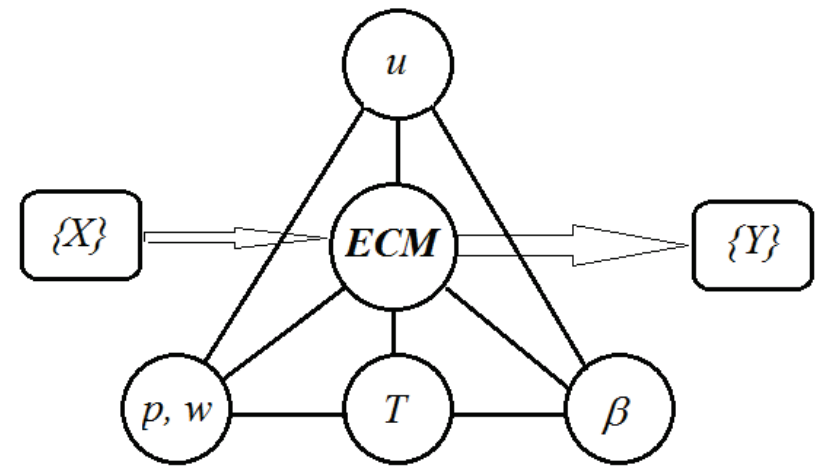

Fig. 2. Schematic diagram of relationships in ECM process [4]

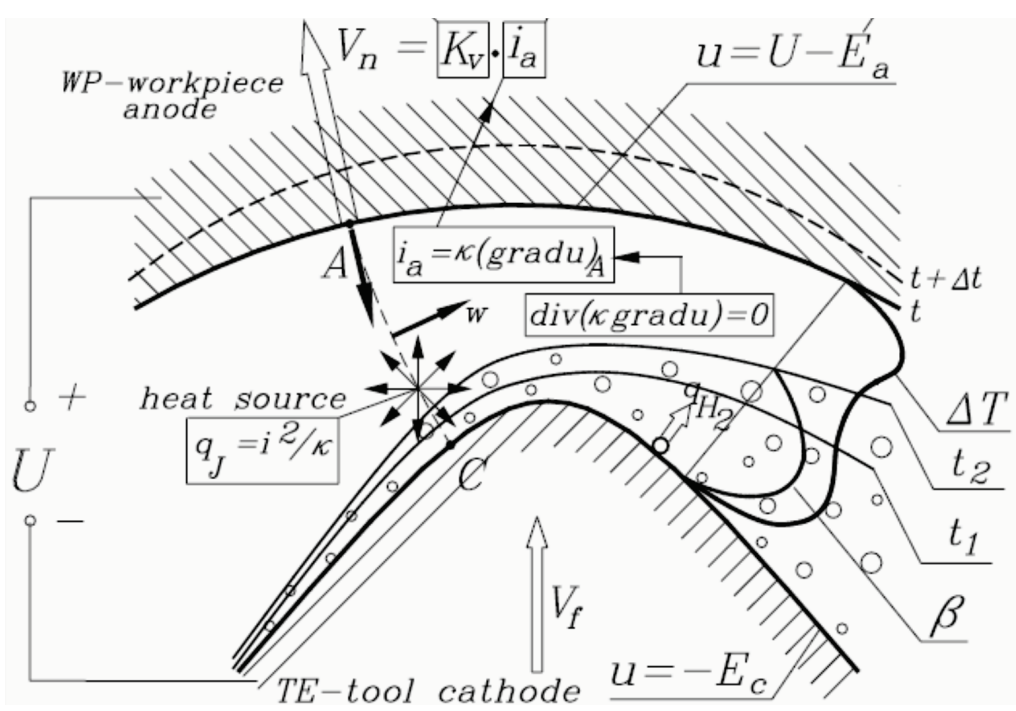

Fig. 3. Schematic diagram of physical state in the gap: $i_{a}$-current density, $U$-voltage, $u$-electrical potential $E_{a}, E_{c}$-over potential on anode and cathode, respectively 
The mathematical model of ECM process, referring to the formulated problems consists of sequence of mutual conjugated partial models, which describe in the gap:

- distribution of the local gap size, $S$,

- distribution of the flow parameters such as the static pressure, $p$, and the velocity, $w$,

- distribution of the temperature, $T$,

- distribution of the void fraction, $\beta$ or the thickness layer, $h$, with two phase flow (electrolyte and gas),

- distribution of the electrical conductivity, $\kappa$.

The more simple mathematical models of the physical conditions and processes in the gap, nevertheless quite adequate in many cases of ECM condition, are the quasi-homogeneous models.

\section{The quasi-homogeneous model of ECM process}

In the quasi-homogeneous models, the multi-phase mixture filling the gap (liquid - gas - solid particle) as well as in layered form, is treated as a uniformly mixed quasi-continuous medium with constant void fraction in the direction normal to the electrolyte flow (see the distribution $\beta$ in Fig. 4) and equal to the void fraction averaged over the cross-section.

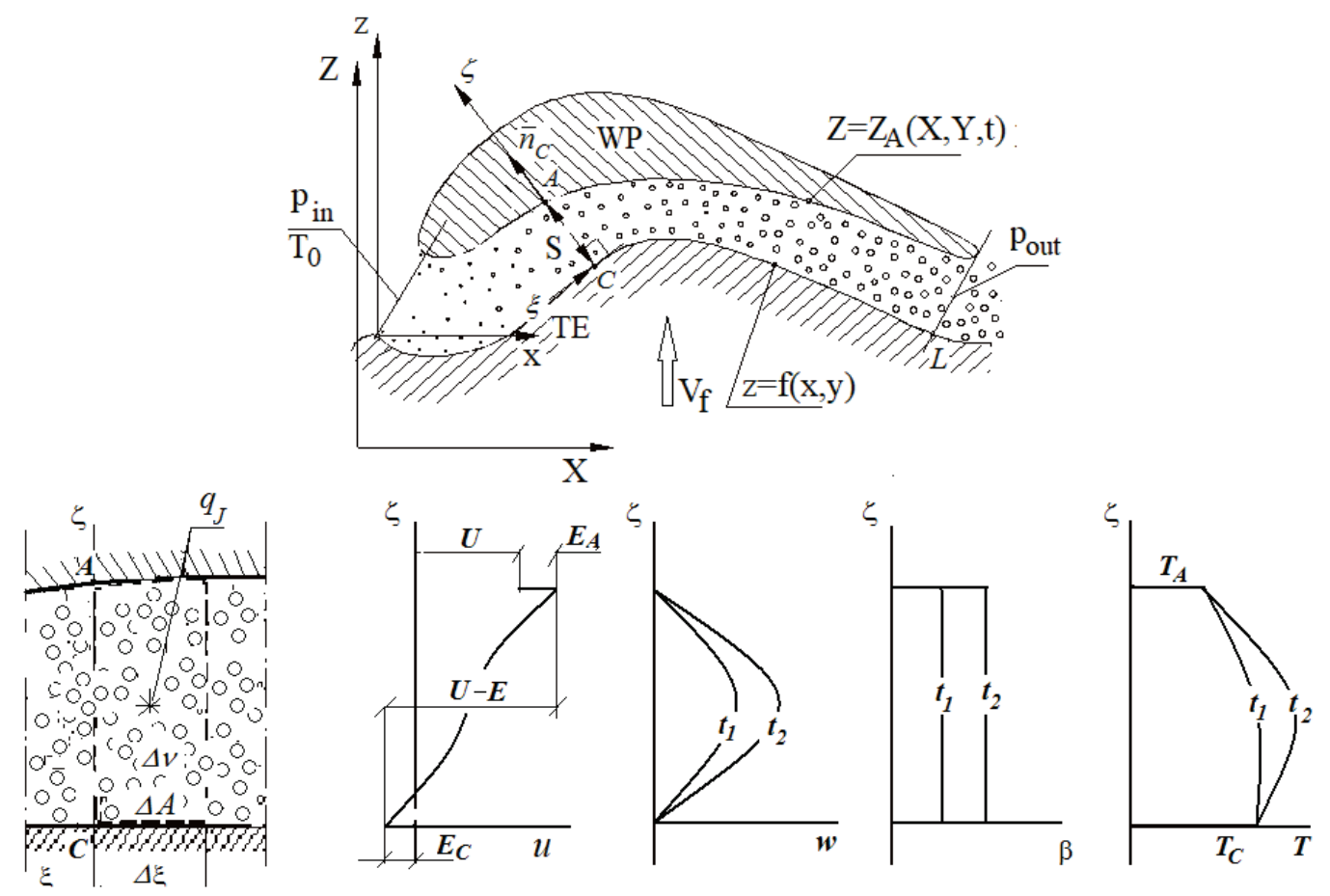

Fig. 4. Schematic of quasi-homogeneous two-phase model of medium in the ECM gap

The following conditions are assumed for above models:

- The bubble layers are assumed to extend completely across the channel.

- Ohm's law is applicable in the whole gap to relate the current density $i$ with medium electrical conductivity $\kappa$, pulse voltage $U$ and gap size $S$.

- The electrical conductive of the two-phase medium can be determined by:

$$
\kappa=\kappa_{o}\left[1+\alpha_{T} \theta\right](1-\beta)^{3 / 2},
$$

where $\alpha_{T}$ is the temperature coefficient of the electrolyte conductivity at $T_{o}, \theta=T-T_{0}$ is temperature increment of electrolyte.

- Electrochemical and diffusion processes are accounted for by the characteristic of anodic dissolution $k_{V}(i)$ and introducing the total over potential $E(i)$ into the boundary condition. 
- The surface tension effect on the gas bubbles is neglected, and so is the bubble formation time.

- The effective density of pseudo-homogeneous medium is determined as following:

$$
\rho=\rho_{e}(1-\beta)+\rho_{g} \beta,
$$

taking into account that liquid density, $\rho_{e}$, is nearly 1000 times more than gases density, $\rho_{g}$, we can set on without loss of accuracy:

$$
\rho=\rho_{e}(1-\beta)
$$

To simplify the calculations let us introduce a curvilinear coordinate system $(\xi, \zeta)$, connected with the tool-electrode (TE) in which a coordinate $\xi$ lies on the given electrode and is measured from the inlet of the electrolyte and let axis $\zeta$ overlap its normal $n_{C}$ (Fig. 4).

To formulate the mathematical model, a general case describing change in shape of the surface of the workpiece can be examined using coordinate system $\{X, Y, Z\}$ attached to the workpiece (WP), which is immovable during machining (Fig. 4). The surface of the workpiece at a given moment in time can be described by: $Z=Z_{A}(X, Y, t)$.

According to electrochemical shaping theory, the evolution of the shape of the workpiece, can be described as follows $[2,4,5]$ :

$$
\frac{\partial Z_{A}}{\partial t}=k_{V}\left(i_{A}\right) i_{A} \sqrt{1+\left(\frac{\partial Z_{A}}{\partial X}\right)^{2}+\left(\frac{\partial Z_{A}}{\partial Y}\right)^{2}},
$$

where: $k_{V}$ is the coefficient electrochemical machinability, which is defined as the volume of material dissolved per unit electrical charge, $i_{A}$ is the current density on the anode.

At the beginning of machining: $t=0, Z=Z_{0}(X, Y)$, where: $Z_{0}(X, Y)$ describes an initial shape of the workpiece surface.

Calculating the current density distribution is generally the crucial step in solving an electrochemical moving boundary problem.

\section{Current density distribution in the gap}

Taking into account that the gap size is much less than the length and the width and the radius of surfaces, we can use one-dimensional approximation for the distribution of electrical potential $u$ along the distance, $S$, of a given point of the anode from the tool-electrode surface is described by equation:

$$
\frac{\partial}{\partial \varsigma}\left(\kappa \frac{\partial u}{\partial \varsigma}\right)=0
$$

with boundary condition: $u(\zeta=0)=0$ and $u(\zeta=S)=U-E$, where $E=E_{a}-E_{c}$ and $E_{a}$, $E_{c}$ are potential of the workpiece - anode and the tool - cathode, respectively.

Integration of Eq. (2) with respect of the assumption, led to:

$$
\kappa_{o}\left[1+\alpha_{T} \theta\right](1-\beta)^{3 / 2} \frac{\partial u}{\partial \varsigma}=i .
$$

After integration with respect of the boundary conditions, is obtained:

$$
i \int_{0}^{S}\left[\kappa_{o}\left(1+\alpha_{T} \theta\right)(1-\beta)^{3 / 2}\right]^{-1} d \varsigma=U-E .
$$

Hence,

$$
i=\kappa_{o} \varphi_{T G} \frac{U-E}{S},
$$


where:

$$
\varphi_{T G}=\left[\frac{1}{S} \int_{o}^{S} \frac{d \zeta}{\left(1+\alpha_{T} \theta\right)(1-\beta)^{3 / 2}}\right]^{-1} .
$$

In one-dimensional mathematical model of ECM, the Eq. (4) is simplified to:

$$
i=\kappa_{o}\left(1+\alpha_{T} \theta\right)(1-\beta)^{3 / 2} \frac{U-E}{S} \text {. }
$$

The averaged across the gap size, values of $\beta$ and $\theta$, is depend on time and coordinate $\xi$.

Distribution of the void faction in the gap

Let us assume, that initial time needed for formation of a gas bubble is much smaller than the machining time, and can be neglected. The equation of mass conservation for the hydrogen generation in the curvilinear coordinates can be obtained from mass balance for the moving control volume, $\Delta v$, in Fig. 3, as:

$$
d\left(\int_{v} \rho_{g} \beta d v\right)=\left(\int_{A(v)} \eta_{H} k_{H} i d A\right) d t .
$$

After transformation, becomes:

$$
\frac{\partial\left(\rho_{g} \beta\right)}{\partial t}+\operatorname{div}\left(w \rho_{g} \beta\right)=\eta_{H} k_{H} i
$$

where: $\rho_{g}=p / R T$ is the specific gas density of hydrogen, $R$ - gas constant for $1 \mathrm{~kg}$ of hydrogen, $\eta_{H}$-current efficiency of the hydrogen generation, $k_{H}$ is the electrochemical equivalent of hydrogen.

In the curvilinear coordinates $(\xi, \zeta)$, the Eq. (7) is obtained the following form:

$$
\frac{\partial(\beta S p / T)}{\partial t}+\frac{(w \beta S p / T)}{\partial \xi}=\frac{10^{5}}{273} \eta_{H} K_{V}^{H} i
$$

with the boundary condition $\beta(0, t)=0$, where $K_{V}^{H}=1.25 \times 10^{-7} \mathrm{~m}^{3} / \mathrm{As}$ is the volume of the hydrogen generated per unit electrical charge $(1 \mathrm{~A} \cdot \mathrm{s})$, at pressure $p=10^{5} \mathrm{~Pa}$ and temperature $T=273 \mathrm{~K}$.

\section{The temperature distribution}

Generally, the temperature distribution in the gap with respect to Joule's heat generation and heat transfer through the electrode surfaces is described by:

$$
\frac{\partial(1-\beta) \theta}{\partial t}+W(\xi, \zeta, t) \frac{\partial(1-\beta) \theta}{\partial \xi}=\frac{\partial}{\partial \zeta}\left[\left(a+a_{T}\right) \frac{\partial(1-\beta) \theta}{\partial \zeta}\right]+\frac{i^{2}}{\kappa_{0} \rho_{e} C_{p}(1-\beta)^{5 / 2}\left(1+\alpha_{T} \theta\right)},
$$

where: $a$-electrolyte thermal diffusivity, $a_{T}$ - turbulent thermal diffusivity by turbulence pulses (for laminar flow $\left.a_{T}=0\right), \rho=\rho_{e}(1-\beta)$ - specific medium density and $C_{p}$ - specific heat of electrolyte, $W(\xi, \zeta, t)$ is a distribution of flow velocity in the gap:

- for laminar flow:

$$
W(\xi, \varsigma, t)=2 w(\xi, t)\left[1-\left(\frac{S-2 \zeta}{S}\right)^{2}\right]
$$


- for turbulent flow:

$$
W(\xi, \varsigma, t)=1.143 w(\xi, t)\left[1-\left|\frac{S-2 \zeta}{S}\right|\right]^{1 / 7}
$$

and $w(\xi, t)$ is averaged velocity across the gap size, which depends on $\xi$ and $t$.

The boundary conditions are as follows: $T(x=0)=T_{0}, T(x, 0)=T_{A}, T(x, S)=T_{C}$; where: $T_{A}$ and $T_{C}$ are temperatures of the anode and cathode, respectively.

\section{Modelling of hydrodynamic conditions in the gap}

To complete the mathematical description, the formulation the pressure and the flow rate must be included.

Taking into account that the working gap thickness is much less than the length and the width, we can write hydrodynamics equations averaged across the gap in direction normal to the toolelectrode surface. Under the given assumptions, we simulate the medium as two-phase homogeneous mixture of an incompressible fluid (water solution of salt and products of the anode reactions) and gas.

Assume that fluid occupies the part $(1-\beta)$ of a small volume of the electrolyte and the part $\beta$ is occupied by the gas. Additional, the mass flow rate of dissolved material is much smaller than the mass flow rate of medium in the gap, and can be neglected in transport equations.

Under given assumptions, the continuity equation can be described in curvilinear system coordinates as follows:

$$
\frac{\partial\left[\rho_{e}(1-\beta) S\right]}{\partial t}+\frac{\partial\left[\rho_{e}(1-\beta) w S\right]}{\partial \xi}=0
$$

Application of the law of motion to the two - phase mixture flowing in the gap i.e. describing of the macroscopic momentum balance equation in the curvilinear coordinates, gives the equation:

$$
\frac{\partial\left[\rho_{e}(1-\beta) S w\right]}{\partial t}+w \frac{\partial\left[\rho_{e}(1-\beta) S w\right]}{\partial \xi}=-S \frac{\partial p}{\partial \xi}-\tau_{A}-\tau_{C},
$$

where $\tau_{A}$ and $\tau_{C}$ are the shear stresses on the surface anode and cathode, respectively, which are assumed to be equal $\left(\tau_{A}=\tau_{C}=\tau\right)$.

In general, the shear stress is expressed by:

$$
\tau=\lambda \rho \frac{w^{2}}{8}
$$

where: $\lambda=C / \operatorname{Re}^{m}$ is friction factor, $\operatorname{Re}=2 w S / v$ is the Reynolds number, for laminar flow i.e. at $\operatorname{Re}<2300: C=96$ and $m=1$, for turbulent flow: $C=0.316$ and $m=0.316$.

The initial conditions is obtained by solution of Eq. (11) at $\beta=0$ and with neglecting the first term of equation, i.e. local derivative on time.

Substituting Eq. (12) into Eq. (11) yields:

$$
\frac{\partial\left[\rho_{e}(1-\beta) S w\right]}{\partial t}+w \frac{\partial\left[\rho_{e}(1-\beta) S w\right]}{\partial \xi}=-S \frac{\partial p}{\partial \xi}-C\left[\frac{v_{e}}{2 w S(1-\beta)^{5 / 2}}\right]^{m} \frac{\rho_{e}(1-\beta) w^{2}}{4} .
$$

The boundary conditions are described by:

$$
p(\xi=0)=p_{\text {in }}-\varsigma_{1} \frac{\rho_{e} w(\xi=0)^{2}}{2}, \quad p(\xi=L)=p_{\text {out }}+\varsigma_{2} \frac{\left[\rho_{e}(1-\beta) w\right]_{\xi=L}^{2}}{2},
$$


where: $\varsigma_{1}, \varsigma_{2}$ are the hydraulic loss pressure in inlet and outlet, respectively.

Basis on presented mathematical models the Simulation Process Module in the computer-aided engineering software CAE-ECM has been developed [4, 7].

\section{An example of computer simulation of electrochemical shaping of blade profile}

To illustrate effects of processes on shaping, the results for machining of a turbine blade are presented.

Computer simulation and tool electrode design has been performed using the CAE-ECM system for following set of input:

- workpiece material - Inconel 817,

- electrolyte $-13 \%$ water solution of $\mathrm{NaNO}_{3}$,

- electrochemical machinability: $k_{v}=1.64-2.13 \exp (-0.03 i), \mathrm{mm}^{3} / \mathrm{Amin}$, where $i$ is a current density in $\mathrm{A} / \mathrm{cm}^{2}$,

- voltage $U=12 \mathrm{~V}$,

- total over potential $E=3 \mathrm{~V}$,

- inlet electrolyte velocity $w=10 \mathrm{~m} / \mathrm{s}$,

- outlet pressure $p_{k}=0.1 \mathrm{MPa}$.

Example of used discretization of 3-D surfaces is shown in Fig. 5 and the initial electrode profiles in Fig. 6.

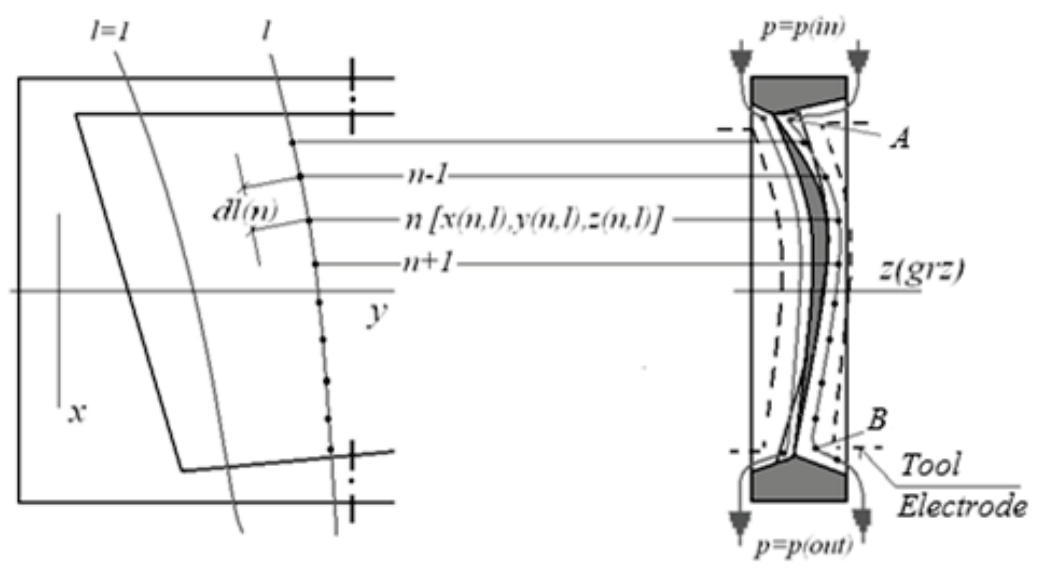

Fig. 5. Discretization of the gap domain along flow lines



Fig. 6. Screen printout containing the electrode profiles in initial stage of ECM 
Examples of simulation of ECM shaping are shown in Fig. 7, where subsequent graphs illustrate anode-workpiece shape evolution in time. Simulation carried out at feed rate $V_{f}=0.8[\mathrm{~mm} / \mathrm{min}]$.

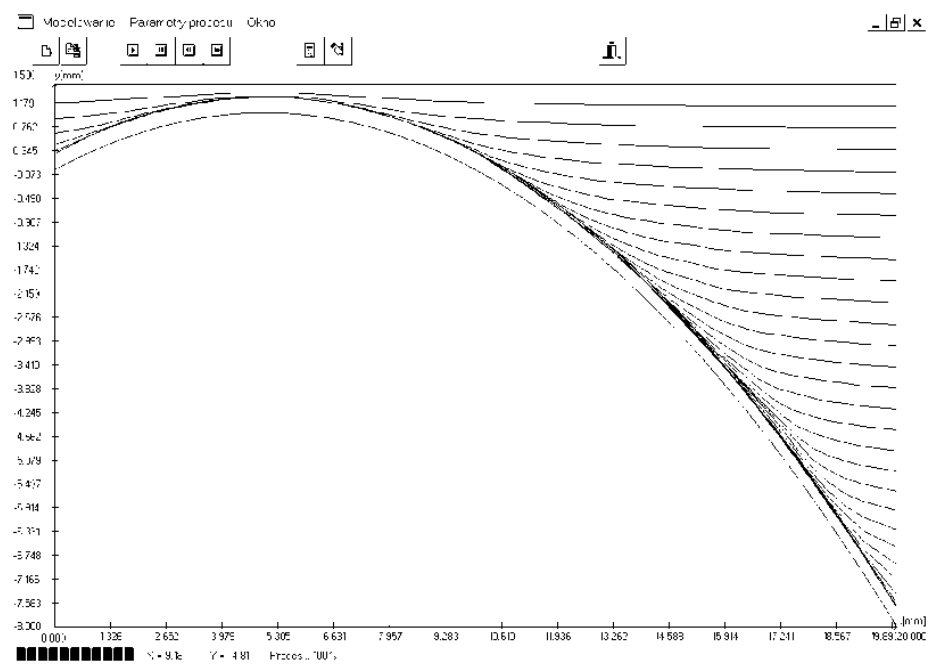

Fig. 7. Screen printout containing the evolution of the shape of the workpiece during machining

Calculated distributions of $S, w, p, T, \beta$ and $i$ as functions of distance along the electrolyte flow (with $x=0$ at the inlet) are shown in (Fig. 8). The change in inter electrode gap width, $S$, results from blade's feather profile change as well as from change of physical conditions along electrolyte flow.

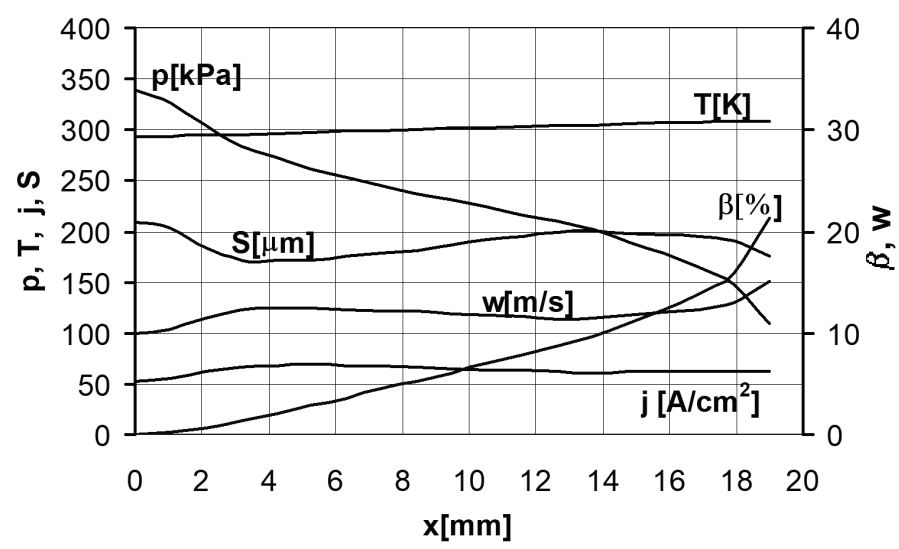

Fig. 8. Calculated distributions of $S, w, p, T, \beta$ and $j$ as functions of distance along the electrolyte flow

To evaluate influence of changes of ECM input parameters on inter electrode gap distribution (or, in other words, on workpiece shape error distribution) the computer simulations for different values of voltage, feed rate and velocity of electrolyte at inlet were performed. Following values for these parameters were used:

- inlet electrolyte velocity $w=5,10$ and $15 \mathrm{~m} / \mathrm{s}$,

- voltage $U=8,12,16 \mathrm{~V}$,

- feed rate $V_{f}=0.75,1.00,1.50 \mathrm{~mm} / \mathrm{min}$.

Some results for these simulations are shown in (Fig. 9-11).

They result from electrolyte a conductivity change that is caused by temperature and gas fraction increase. Decrease of inlet velocity of electrolyte causes decrease of gap width at the electrolyte outlet. Gap width significantly depends on pressure at the outlet what can be seen from graphs in (Fig. 9) for $p_{k}=0.10 \mathrm{MPa}$ and $p_{k}=0.15 \mathrm{MPa}$. 
Increase of outlet pressure causes significant increase of gap width what can be explained by decrease of concentration of gas phase.

Changes of profiles with respect to reference profile, $b l p 5$, for different electrolyte velocities are shown in (Fig. 9).

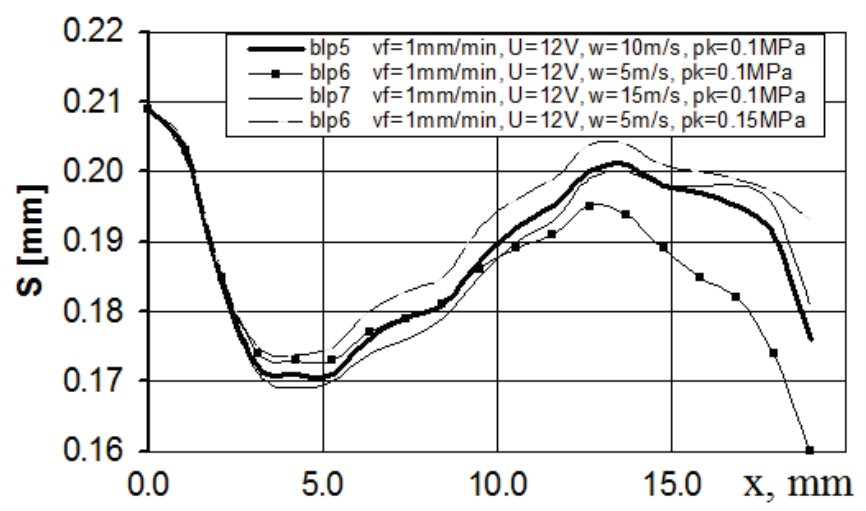

Fig. 9. The gap width for different input parameters

Despite significant changes of inlet velocity, the maximum difference between profiles was relatively small $(<0.03 \mathrm{~mm})$. Much more pronounced influence on the machined profile has working voltage, $U$, and electrode feed rate, $V_{f}$.

As an example, influence of feed rate on shape deviation is shown in (Fig. 10).

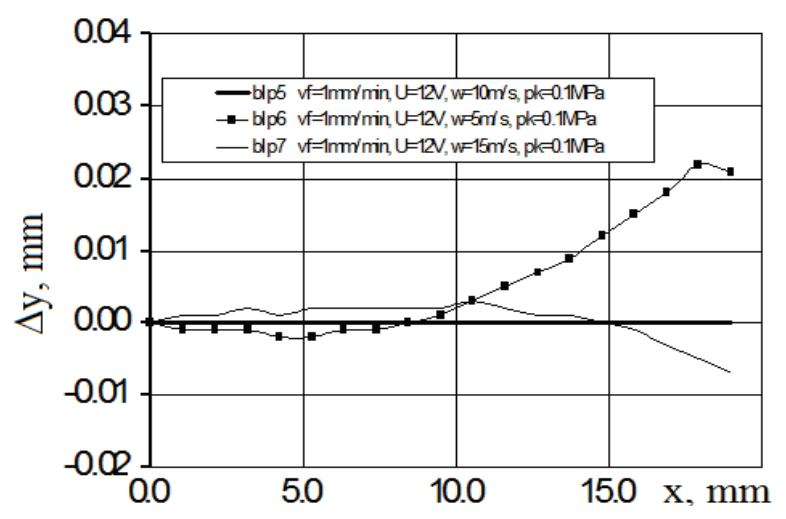

Fig. 10. Distribution of profile deviations with respect to reference profile blp5 for different electrolyte velocities

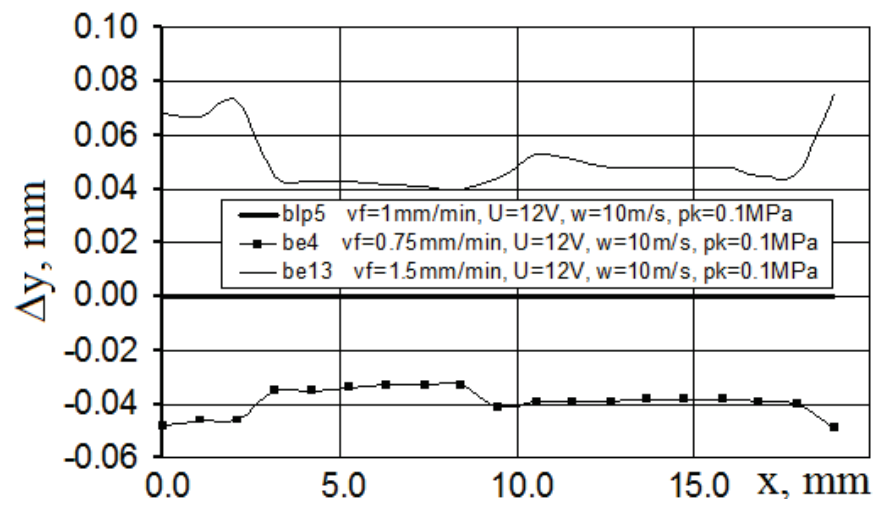

Fig. 11. Distribution of profile deviations with respect to reference profile blp 5 for different electrode feed rates

Results show that, for reducing the profile deviations, it is necessary stabilization of these parameters during machining. Their values should be maintained as close as possible to their nominal values meaning values that were used for tool electrode design. 


\section{Improvement of machining accuracy by using pulse current}

The analyses led to the conclusion, that machining inaccuracy is proportional to the gap size. Therefore, for improvement of shape accuracy and simplification of tool design, the gap size during ECM should be smaller as possible. Additionally, more stables the gap state is needed by reduce non-uniformity of electrical conductivity and other physical conditions, which are significant for dissolution process.

All these requirements for ECM performance, with continuous working voltage are very limited. The minimum practical tool gap size, which may be employed, however is constrained by the onset of unwanted electrical discharges. All these constrain of continuous ECM can be eliminated and the requirements with view of points of machining accuracy can be achieved by application of pulse working voltage $[2,4,5,7,8]$.

In the pulse electrochemical machining (PECM) process, a pulse generator is used to supply the working voltage pulses across the two electrodes, typically in the form of pulse strings consisting of single pulses or grouped pulses (Fig. 12).
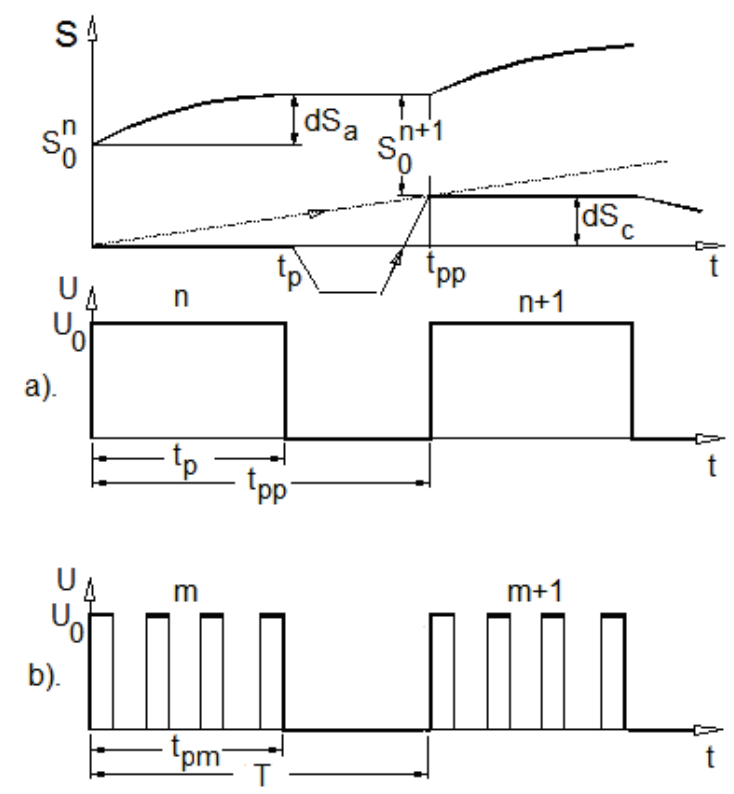

Fig. 12. A scheme of PECM using: a) serial of single voltage pulses, b) serial of pockets with single voltage pulses

The anodic electrochemical dissolution occurs during the short pulse on-times $t_{p}$, each ranging from $0.1 \mathrm{~ms}$ to $5 \mathrm{~ms}$. Dissolution products (sludge, gas bubbles and heat) are flushed away from the inter-electrode gap by the flowing electrolyte during the pulse off-times between two pulses or two groups of pulses. To intensify the electrolyte flushing, the tool is retracted from the workpiece to enlarge the gap during the pulse off-times. The gap checking and tool repositioning can also be conducted during these pulse pauses to establish a given gap size before the arrival of the next pulse, leading to a significant reduction in the indeterminacy of the gap and, hence, of the shaping accuracy.

In the result, the continuous ECM is replaced with discrete process, which makes possible to reduce the gap size below $0.1[\mathrm{~mm}]$, and to set the gap size intermittently by controlling (monitoring) the position of the machining surface during the pause between pulses.

Practice and investigations shows that introducing of PECM allows:

- diminish the inter-electrode below $0.1 \mathrm{~mm}$, what is one of the fundamental condition of increasing ECM accuracy,

- reduce the inaccuracy of the machined profile caused by internal disturbance of physical and chemical properties of electrolyte (more exact: medium in the gap) in inter-electrode gap, 
- simplify of tool design since the much more uniformity distribution of the gap size,

- eliminate the macro-defects on the machined surface connected with the hydrodynamics flow disturbance,

- monitoring and control the gap sizes on line i.e. during machining cycle.

Disadvantages of PECM is decreasing average feed rate in comparison to continuous ECM. Therefore, application of PECM for removing all allowance usual consuming large time. For solve this problem can be used the sequence of ECM and PECM processes performed on one machine tool. After ECM stage of shaping, when basic amount of material is removed (approx. 80\%) and rough shape with accuracy about $0.1-0.3 \mathrm{~mm}$ is obtained, the PECM stage is applied for machining final shape with high accuracy. Main an advantage of this method is very significant reduction of machining time, because material removal in ECM stage is more than 5-10 times larger than PECM.

The sequence ECM-PECM has been used in machining of airfoils as shown in Fig. 13.

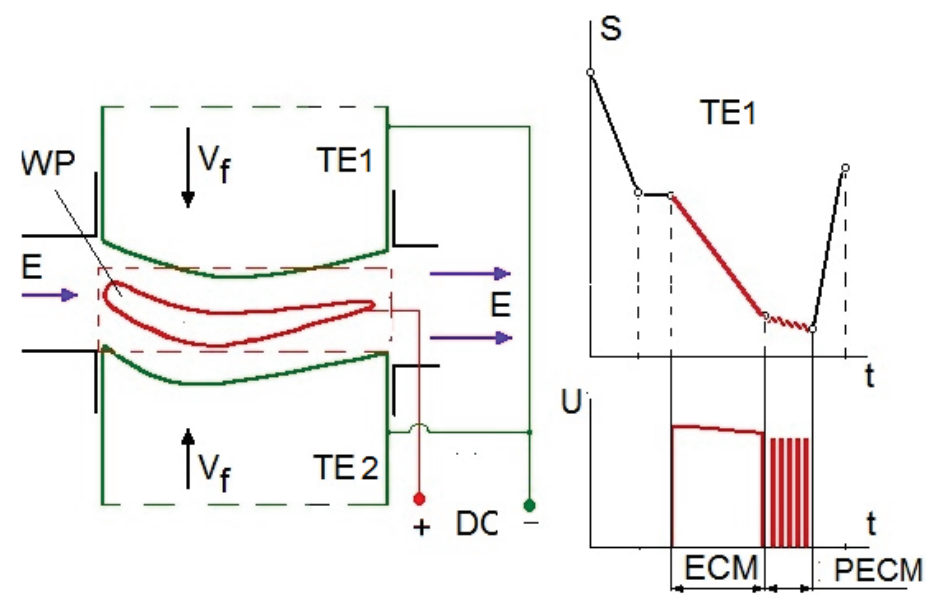

Fig. 13. Principal scheme of the shaping of both side of airfoil with using the sequences of ECM and PECM treatments

$T E(1,2)$ - tool electrodes, WP - workpiece, E-electrolyte, DC-power supply of continuous and pulse current

During the stage of ECM, the frontal gap is reached of the stationary gap $S_{f}$, which is initial frontal gap for PECM process. In the final moment of PECM, the frontal gap size is equal of $A C(p)$ (Fig. 14).

The optimal conditions of shaping from the point of view of accuracy and productivity are determined by computer simulation of sequence of both processes (ECM and PECM).

Figures from 15 to 17 shows of the selected results of computer simulation of airfoil shaping at following conditions: material of workpiece, $\gamma \mathrm{TiAl}$; electrolyte $15 \% \mathrm{NaCl}$, working voltage, $U=15 \mathrm{~V}$; pulse on time, $t_{p}=2 \mathrm{~ms}$; pulse off time $t_{p p}=5.0 \mathrm{~ms}$; displacement of tool electrode after each pocket (Fig. 11) $d S_{C}=0.005 \mathrm{~mm}$; number of pulses in the pocket $N=115$; number of pockets $M=150$; pocket period $T=1 \mathrm{sec}$.

The changes of current densities and increments temperature during rectangle voltage pulse are shown in Fig. 15. During all PECM treatment, thermal conditions in the gap were below limitation.

The evolution in time of the local gap size along airfoil profile i.e. at different angle, $\alpha$, has been determined using the computer simulation. Example of changes gap size in time is shown in Fig. 16. After some number of pockets pulses, the gap size practically stabilizes on a quasi-steady state value, $S_{n}$.

The shape error of airfoil is depend on deviation of gap size along profile, which can be calculated as following:

$$
\Delta S_{n}=S_{n}(\alpha)-S_{n}(\alpha=0)
$$


Figure 17 illustrated of the distribution of the value $\Delta S_{n}$ along airfoil profile in quasi-steady state of PECM and steady ECM.

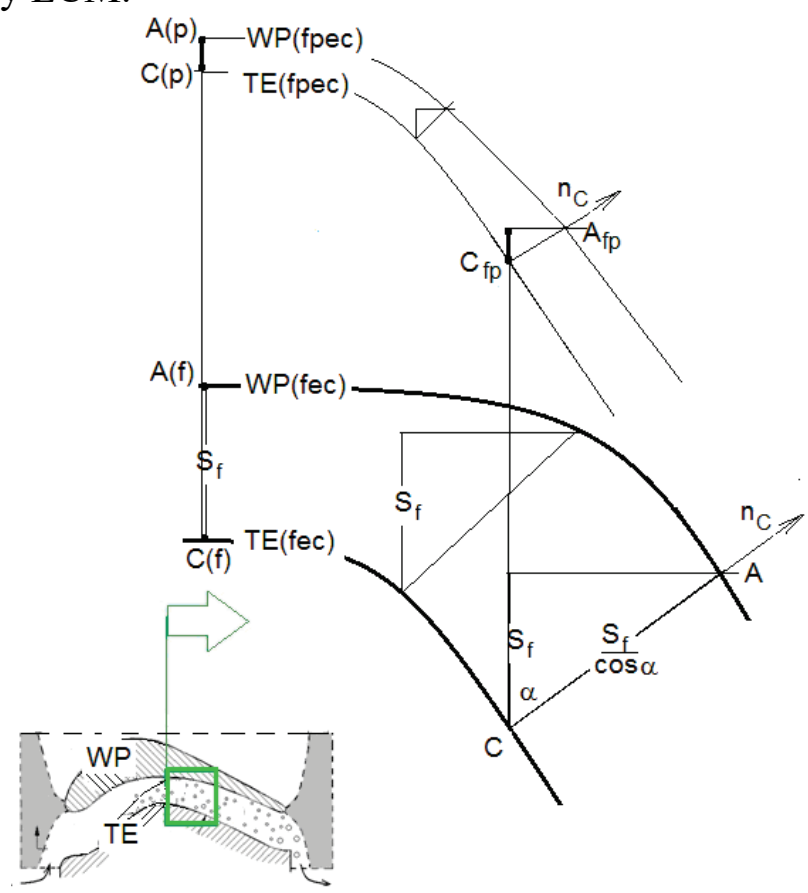

Fig. 14. Scheme of the position of electrodes: TE(fec) and WP(fec) - the position of the tool electrode and machined profile respectively, in the final moment of ECM and the initial moment of PECM

$T E(f p e c), W P(f p e c)$ - the position of TE and WP in the final moment of PECM

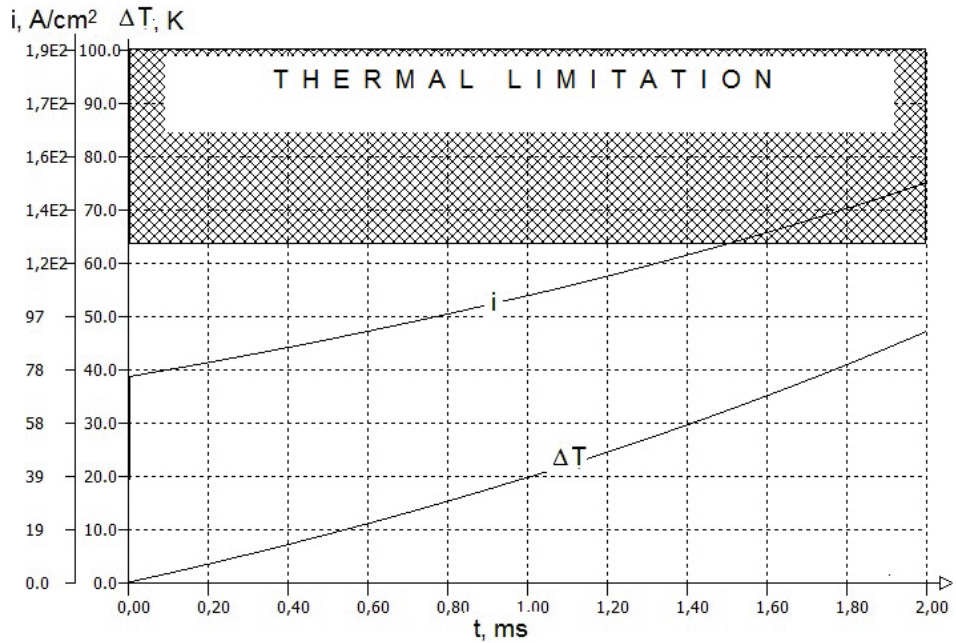

Fig. 15. The current density and the electrolyte temperature changes during pulse on - time in last pulse $(n=115, m=150)$



Fig. 16. Changes in the local gap size at different angle during serial pulse pocket $M$ 


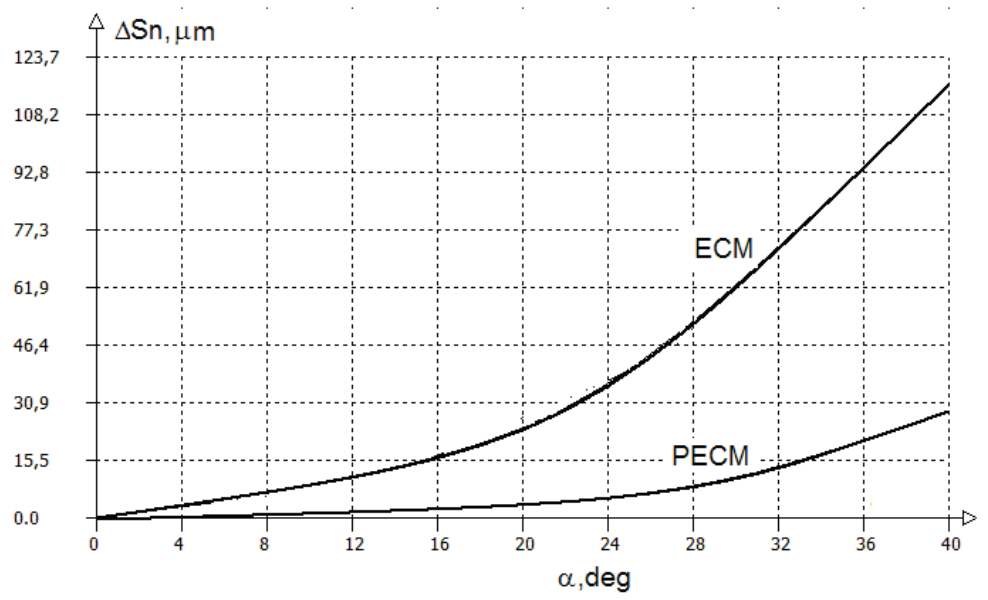

Fig. 17. The distributions of the value of deviation $\triangle S_{n}$ in ECM and PECM processes

The results of simulation presented in Fig. 17, shows that the gap size distribution in the PECM process is more uniform than that in the continuous ECM, These conclusions has been confirmed by experiments and practice of PECM. It is reasonable to treat the gap distribution as equidistant in the PECM

The practice of cooperation with industry shows that the shaping of airfoils using the sequences ECM-PECM processes is an effective way for improve of quality and productivity of ECM technology.

\section{References}

[1] Davydov, A. D., Volgin, V. M., Electrochemical Machining, Encyclopedia of Electrochemistry, Vol. 5, Chapter 12, Electrochemical Engineering, Bard, A. J. ed., Willey-VCH, New York 2007.

[2] Davydov, A. D., Kozak, J., High Rate Electrochemical Shaping, Ed. Nauka, Moscow 1990.

[3] Flightpath 2050 (2011) Europe's Vision for Aviation, Report of the High-Level Group on Aviation Research, Publications Office of the European Union Luxembourg, 2011.

[4] Kozak, J., Mathematical Modelling of Advanced Manufacturing Processes, Scientific Library of Institute of Aviation, No. 56, Warsaw 2019.

[5] Kozak, J., Surface Shaping by Electrochemical Machining, Transaction of Warsaw University of Technology, Warsaw 1976.

[6] Kozak, J., Computer Simulation System for Electrochemical Shaping, Journal of Materials Processing Technology, Vol. 109, No. 3, pp. 354-359, 2001.

[7] Kozak, J., Lubkowski, K., The Basic Investigation of Characteristic in the Pulse ECM, Proceed. 20 ${ }^{\text {th }}$ M.T.D.R. Int. Conf., pp. 625-630, Birmingham 1979.

[8] Kozak, J., Rajurkar, K. P., Wei, B., Modelling and Analysis of Pulse Electrochemical Machining (PECM), Transaction of ASME - Journal of Engineering for Industry, Vol. 116, No. 3, 1994.

[9] Purcar, M., Dorochenko, A., Borteis, L., Deconinck, J. B., Van den Bossche, B., Advanced $C A D$ integrated approach for $3 D$ electrochemical machining simulations, Journal of Materials Processing Technology, Vol. 203, pp. 58-71, 2008.

[10] Rajurkar, K. P., McGeough, J. A., Kozak, J., De Silva, A., New Developments in ElectroChemical Machining, Annals of the CIRP, Vol. 48/2, pp. 567-579, 1999.

Manuscript received 17 June 2019; approved for printing 23 September 2019 